\title{
A Two-Step Compressed Sensing Approach for Single-Snapshot DOA Estimation of Closely Spaced Signals
}

\author{
Shuang Wei $(\mathbb{D}$, Yanhua Long $(\mathbb{D}$, Rui Liu, and Ying Su \\ College of Information, Mechanical and Electrical Eng, Shanghai Normal University, Shanghai, China \\ Correspondence should be addressed to Yanhua Long; yanhua@shnu.edu.cn
}

Received 22 June 2020; Revised 12 December 2020; Accepted 13 January 2021; Published 2 February 2021

Academic Editor: Ramon Sancibrian

Copyright (๑) 2021 Shuang Wei et al. This is an open access article distributed under the Creative Commons Attribution License, which permits unrestricted use, distribution, and reproduction in any medium, provided the original work is properly cited.

Single-snapshot direction-of-arrival (DOA) estimation plays an important role in dynamic target detection and tracking applications. Because a single-snapshot signal provides few information for statistics calculation, recently compressed sensing (CS) theory is applied to solve single-snapshot DOA estimation, instead of the traditional DOA methods based on statistics. However, when the unknown sources are closely located, the spatial signals are highly correlated, and its overcomplete dictionary is made up of dense grids, which leads to a serious decrease in the estimation accuracy of the CS-based algorithm. In order to solve this problem, this paper proposed a two-step compressed sensing-based algorithm for the single-snapshot DOA estimation of closely spaced signals. The overcomplete dictionaries with coarse and refined grids are used in the two steps, respectively. The measurement matrix is constructed by using a very sparse projection scheme based on chaotic sequences because chaotic sequences have determinism and pseudo-randomness property. Such measurement matrix is mainly proposed for compressing the overcomplete dictionary in preestimation step, while it is well designed by choosing the steering vectors of true DOA in the accurate estimation step, in which the neighborhood information around the true DOAs partly solved in the previous step will be used. Monte Carlo simulation results demonstrate that the proposed algorithm can perform better than other existing single-snapshot DOA estimation methods. Especially, it can work well to solve the issues caused by closely spaced signals and single snapshot.

\section{Introduction}

Direction-of-arrival (DOA) estimation plays an important role for target/source localization, which is widely used in many fields including radar, sonar, speech, communication, and medical diagnosis [1-4]. Traditional high-resolution DOA estimation algorithms use the statistics of observed signals to improve the performance efficiency, such as multiple signal classification (MUSIC) algorithm and estimation method of signal parameters via rotational invariance techniques (ESPRIT) [5,6]. These algorithms require to receive the signals observed in a period of time. However, in dynamic target detection and tracking systems, only single snapshot or a small number of snapshots are available for DOA estimation. In this case, the statistics information is not accurate, and thus traditional algorithms degrade dramatically. Therefore, single-snapshot DOA estimation attracts much attention, which is an active topic widely used in automotive radar/sonar applications like driver assistance systems [7]. Moreover, Häcker and Yang [7] also investigated the performance of traditional DOA estimators using a single snapshot, such as Bartlett beamformer, MUSIC, deterministic maximum likelihood, stochastic maximum likelihood, and weighted subspace fitting. It was shown that these algorithms cannot work at all or cannot show a superior performance as expected, especially for multitarget complex scenarios including correlated targets under low SNR situation.

In order to improve the accuracy of single-snapshot DOA estimation, the latest research studies on this topic mainly used the compressed sensing theory $[8,9]$ because in CSbased algorithms, the signals can be reconstructed in spatial by an overcomplete dictionary, whose sampling interval could be smaller than the Nyquist limit [10]. However, considering that the unknown angles are closely spaced, an overcomplete 
dictionary is composed of a uniform sampling grid, so that the on-grid points should be densely distributed to cover all the exactly steering vectors to reduce the gap between the real DOA and its nearest grid points. That means vectors involving true DOAs in the overcomplete dictionary will be high dimensional, and computational cost will be high in sequence. Furthermore, the columns in the matrix with high correlation will inevitably degrade the estimation performance. Therefore, the design of overcomplete dictionary becomes the key step in the CS-based single-snapshot DOA estimation algorithms for closely spaced signals, which is also the main content of this paper.

Many researchers focus on the design of overcomplete dictionary to improve the accuracy of CS-based estimators. Some existing CS-based estimators have designed iterative schemes to obtain the optimal overcomplete dictionary [11-13]. Some researchers designed the approach based on off-grid model which can achieve a smaller MSE than those methods based on on-grid model. Among them, some off-grid methods require the covariance matrix of multiple observed samples, such as the sparse asymptotic minimum variance (SAMV) method in [14]. However, in some dynamic applications, the statistics of multiple previous snapshots are inconsistent with the characteristics of the current single snapshot. Some methods have been developed based on Bayesian compressed sensing framework to model the off-grids in the overcomplete dictionary, such as the off-grid sparse Bayesian inference (OGSBI) method in $[15,16]$. Some DOA estimation methods use the total least-squares to reduce the error of the overcomplete dictionary, such as [17]. One drawback of these methods is its slow speed in the case of a dense sampling grid. When the estimated DOA is densely distributed, the iterative algorithms based on the probabilistic model need to spend a large amount of computational load to maintain the estimation accuracy.

In order to improve the estimation accuracy, this paper proposed a two-step method to construct the overcomplete dictionary. In the first step, the search space with coarse grids will be used, which is useful to determine a narrow optimal range of the second step with a reduced computational cost. Thus, high correlation of the vectors will be reduced to a low level, and the key neighborhood information around the true DOAs lies in the preconceived results [15]. In the following step, the previous result will be used to reconstruct the overcomplete dictionary with a smaller size, which is useful to reduce the memory cost. Then, an updated search space with refined grids will be used, so that a more accurate DOAs estimation can be expected with high probability in the final step.

Besides, in order to obtain the sparse angles from a single-snapshot signal, a measurement matrix is required to sample the observed signals without loss of information [18]. The measurement matrix has to meet the restricted isometry property (RIP) to ensure the completeness of useful information [19-22]. Generally, Gaussian random matrix and Bernoulli matrix are used to construct the measurement matrix because it is found $[23,24]$ that they can perform better with small measurement number than other matrices. However, their randomness and high memory cost are two issues in application. In order to solve this issue, some alternate minimization methods were proposed to design the optimal measurement matrix in terms of reducing the coherence between the atoms of such matrix [25-29]. The optimization target is to minimize the difference between the Gram matrix of the equivalent dictionary and the identity matrix [30]. The equivalent dictionary is defined as the product of the measurement matrix and the overcomplete dictionary. Thus, in this paper, a new double-structure measurement matrix is constructed by combining a part of the unit matrix and the chaotic measurement matrix because the chaotic measurement matrix has been proved to satisfy the RIP [22, 31, 32] and has better properties than Gaussian matrix [33]. In addition, the very sparse random projection (VSRP) method is used to thin the chaotic matrix, in order to reduce the memory cost, especially when the measurement number is large.

The main contribution of this paper is to develop a novel approach for single-snapshot DOA estimation of closely spaced signals. Its contributions are twofold.

We proposed a two-step compressed sensing approach to improve the estimation accuracy by decreasing high correlation in the overcomplete dictionary. In the first step, the search space with coarse grids is defined, so that high correlation of the vectors will be reduced to a low level and high computation cost will be avoided. In the second step, an updated search space with refined grids is designed, which is adaptively determined by the solution obtained from the first step. Subsequently, a smaller dictionary set defined around the true DOAs lies in the preconceived results, which can improve the single-snapshot DOA estimation efficiency.

The proposed CS-based method used a double-structure measurement matrix for closely spaced DOA estimation. In order to minimize the difference between its Gram matrix and the identity matrix, a part of the unit matrix and a chaotic-based measurement matrix are combined to construct this double-structure measurement matrix. The chaotic-based measurement matrix is built by logistic mapping chaotic sequence and very sparse random projection method, where very sparse projection scheme is used to thin the chaotic matrix. Experimental results show that it significantly improves the estimation accuracy of CS-based method and outperforms those obtained using the general Gaussian random matrix.

The remainder of the paper is organized as follows. Section 2 describes a single-snapshot DOA estimation model using CS theory. Section 3 presents the proposed double-structure measurement matrix designed by chaotic sequence and very sparse random projection method. The proposed two-step CS-based method for DOA estimation is then presented in Section 4 by using the proposed measurement matrix. In Section 5, some numerical experiments are given by using dense spatial source signals, and the results are analyzed from the perspective of different measurement numbers, SNRs, and sparse degrees. Conclusions and future works are provided in Section 6. 


\section{One-Step DOA Estimation of a Single Snapshot}

Consider $K$ narrow-band signals from the unknown directions $\theta_{i}(i=1,2, \ldots, K)$ incidenting to uniform linear array (ULA) composed of $N$ sensors with interelement spacing $d$; then, the observed output at a single snapshot $\mathbf{x} \in \mathbb{C}^{N \times 1}$ is expressed as [34]

$$
\mathbf{x}=\mathbf{A s}+\mathbf{w}
$$

where $\mathbf{s} \in \mathbb{C}^{K \times 1}$ is the signal vector, $\mathbf{w} \in \mathbb{C}^{N \times 1}$ is the additive Gaussian white noise, and $\mathbf{A} \in \mathbb{C}^{N \times K}$ is the array manifold matrix, which is given as $[35,36]$

$$
\mathbf{A}=\left[\mathbf{a}\left(\theta_{1}\right), \mathbf{a}\left(\theta_{2}\right), \ldots, \mathbf{a}\left(\theta_{K}\right)\right],
$$

where $\mathbf{a}\left(\theta_{i}\right)=\left[\begin{array}{llll}1 & e^{-j(2 \pi / \lambda) d \sin \theta_{i}} & \ldots & e^{-j(2 \pi / \lambda)(N-1) d \sin \theta_{i}}\end{array}\right]^{T}$ with $i=1,2, \ldots, K$ is the steering vector and $\lambda$ is the wavelength.

Consider the angular search range of interest $\Theta$ involving from $\theta_{b}$ to $\theta_{e}$, which is defined by

$$
\Theta=\left[\begin{array}{llll}
\bar{\theta}_{1} & \bar{\theta}_{2} & \ldots & \bar{\theta}_{L}
\end{array}\right]^{T},
$$

with the candidate angles defined by $\bar{\theta}_{j}=\theta_{b}+(j-$ $1 / L) \times\left(\theta_{e}-\theta_{b}\right)(j=1,2, \ldots, L) . L$ is the number of the uniform-distributed candidate angles, and it has relation $L \gg K$. Thus, the overcomplete array manifold matrix $\Psi \in \mathbb{C}^{N \times L}$ composed of the steering vectors associating with each angle orientation $\bar{\theta}_{j}$ is expressed as

$$
\boldsymbol{\Psi}=\frac{1}{\sqrt{L}}\left[\mathbf{a}\left(\bar{\theta}_{1}\right), \mathbf{a}\left(\bar{\theta}_{2}\right), \ldots, \mathbf{a}\left(\bar{\theta}_{L}\right)\right]
$$

where $\mathbf{a}\left(\bar{\theta}_{j}\right)=\left[\begin{array}{llll}1 & e^{-j 2 \pi\left(\mathrm{d} \sin \bar{\theta}_{j} / \lambda\right)} & \ldots & e^{-j 2 \pi(N-1)\left(\mathrm{d} \sin \bar{\theta}_{j} / \lambda\right)}\end{array}\right]^{T}$ $\in \mathbb{C}^{N \times 1}$ is the steering vector corresponding to each angle orientation $\bar{\theta}_{j}$. So, equation (1) can be rewritten as [37]

$$
\mathbf{x}=\Psi \mathbf{y}+\mathbf{w}
$$

where $\mathbf{y}=\left[\begin{array}{llll}y_{1} & y_{2} & \ldots & y_{L}\end{array}\right]^{T} \in \mathbb{C}^{L \times 1}$ denotes the candidate signal component corresponding to all $L$ possible DOAs. Because only true(unknown) DOAs have the larger components than the other angles, $\mathbf{y}$ can be considered a $K$-sparse vector. That is, $\mathbf{y}$ is a sparse vector to be solved, which has only $K$ larger components and the rest $L-K$ is close to zero.

Based on CS theory, a measurement matrix $\Phi \in \mathbb{C}^{M \times N}$ is designed to measure the original output $\mathbf{x}$, and a measured signal $\mathbf{z} \in \mathbb{C}^{M \times 1}$ is obtained as [37]

$$
\mathbf{z}=\Phi \mathbf{x}=\Phi(\Psi \mathbf{y}+\mathbf{w})
$$

where $M$ is the measurement numbers and $M<N$. According to CS theory, as long as the measurement number $M$ satisfied the following equation, i.e., $M \geq K \times \log _{2}(N / K)$, the vector $\mathbf{y}$ can be obtained accurately with a big probability [38]. In equation (6), the measurement matrix $\Phi$ is commonly constructed by random sequences which can let the columns of the equivalent dictionary have low correlations with each other, such as Gaussian random matrix. However, its randomness and high memory cost are two issues that need to be solved in application. Thus, we proposed a double-structure measurement matrix in Section 3.

The sparse characteristic of $\mathbf{y}$ shown in equation (6) can be considered as a constraint condition [39], so that the vector $\mathbf{y}$ in equations (5) and (6) can be solved by a $l_{1}$ norm optimization problem as [37]

$$
\begin{aligned}
& \min \|\widehat{y}\|_{1} \\
& \text { s.t. }\|\mathbf{z}-\boldsymbol{\Phi} \Psi \hat{y}\|_{2}<\varepsilon,
\end{aligned}
$$

where $\hat{y}$ denotes the estimated sparse solution and $\varepsilon$ is a small parameter. Thus, the estimated DOAs of the spatial signals can be decided by the nonzero position of the sparse vector $y$ after such vector $\mathbf{y}$ is solved according to the measured vector z. In summary of the above, a one-step CS-based algorithm for a single snapshot is shown in the following (Algorithm 1).

\section{Proposed Measurement Matrix Based on Very Sparse Chaotic Sequences}

Considering a chaotic measurement matrix constructed by logistic chaotic sequences, the mathematical expression of mapping equation is [24]

$$
x_{g+1}=\mu x_{g}\left(1-x_{g}\right), \quad n=0,1,2 \ldots, G,
$$

where $x_{g} \in(0,1)$ represents the $g^{\text {th }}$ value of chaotic sequence and $g$ denotes the iteration number. In the case of $\mu=4$, the values of $x_{g}$ can traverse the entire area of 0 to 1 , and each point of the sequences has the property of pseudo-randomness $[31,33]$. So, the proposed method defines $\mu=4$. The initial value is set as $x_{0}=0.256$.

In order to improve the stability, after generating $x_{0}, x_{1}, \ldots, x_{G}$, the first $t$ items are discarded to form a new sequence $x_{t}, x_{t+1}, \ldots, x_{G}$. The chaotic sequence is sampled by the equal interval of $v$, that is,

$$
z_{\sigma}=x_{t+\sigma v}
$$

where $v$ denotes the sampling rate of the sequence $x$. The pseudorandom sequence $z_{0}, z_{1}, \ldots, z_{(G-t) / v}$ is then obtained. In our experiments, $G$ is set to $3000 \times 3000, v$ is set to 3 , and $t$ is set to 1000. Because Yu and Barbot et al. [24] have proved that a matrix constructed by a chaotic sequence column by column is sufficient to satisfy RIP with high probability, the first $M \times M$ values are selected to generate a chaotic matrix $\Gamma \in \mathbb{R}^{M \times M}$ with $M$ being the measurement number:

$$
\Gamma=\left[\begin{array}{cccc}
z_{0} & z_{M} & \ldots & z_{M \times(M-1)} \\
z_{1} & z_{M+1} & \ldots & z_{M \times(M-1)+1} \\
\ldots & \ldots & \ldots & \ldots \\
z_{M-1} & z_{2 M-1} & \ldots & z_{M \times M-1}
\end{array}\right] .
$$

When the number of measurements $M$ is large, the memory cost of chaotic matrix $\Gamma$ shown in equation (10) would increase greatly. In order to compress $\Gamma$ without the loss of useful information, very sparse random projection (VSRP) method is applied to the chaotic measurement 
Input:
The observed single-snapshot signal $\mathbf{x} \in \mathbb{C}^{N \times 1}$;

The observed single-snapshot signal $\mathbf{x}$
The measurement matrix $\Phi \in \mathbb{C}^{M \times N}$;

Output:

The DOA estimator, $\left[\phi_{1}, \phi_{2}, \ldots, \phi_{K}\right]$;

(1) Divide the initial search space $\Theta$ into $L$ parts to construct the overcomplete dictionary matrix $\Psi \in \mathbb{C}^{N \times L}$.

(2) Generate the measured signal $\mathbf{z}=\Phi \mathbf{x}$ and the sensing matrix $\mathbf{T}=\Phi \Psi$.

(3) Calculate the DOA estimator $\left[\phi_{1}, \phi_{2}, \ldots, \phi_{K}\right]$ using the OMP method in [40].

(4) return $\left[\phi_{1}, \phi_{2}, \ldots, \phi_{K}\right]$.

Algorithm 1: The one-step CS-based algorithm.

matrix [32], and it is also used to reduce the data recorded in the memory [41] and make the measurement matrix easy to implement [42].

In fact, very sparse projection is the generalized form of sparse projection and still satisfies the sparse projection distribution as

$$
\Phi_{1}(i, j)= \begin{cases}\sqrt{S}, & \text { with prob. } \frac{1}{2 S} \\ -\sqrt{S}, & \text { with prob. } \frac{1}{2 S} \\ 0, & \text { with prob. } 1-\frac{1}{S},\end{cases}
$$

where in the case of $1 \leq S \leq 3$ every element in $\Phi_{1}$ meets the sparse distribution, while in the case of $S \gg 3$ every element in $\Phi_{1}$ meets the very sparse distribution [43]. Inspired by this, we propose a very sparse projection scheme $\Phi_{2}$ to improve the chaotic measurement matrix as follows:

$$
\Phi_{2}(i, j)= \begin{cases}\sqrt{M}, & \frac{1}{\sqrt{M}}>\Gamma(i, j) \geq 0, \\ -\sqrt{M}, & \frac{2}{\sqrt{M}}>\Gamma(i, j) \geq \frac{1}{\sqrt{M}}, \\ 0, & 1>\Gamma(i, j) \geq \frac{2}{\sqrt{M}} .\end{cases}
$$

Here, $\Phi_{2} \in \mathbb{R}^{M \times M}$ is the matrix obtained by thinning the chaotic matrix $\Gamma$, and $\Phi_{2}$ has similar characteristics with $\Phi_{1}$. Since very sparse matrix has been proved to meet the RIP $[32,43], \Phi_{2}$ satisfies the RIP theory as well.

According to the CS theory, a measurement matrix composed of two different structural matrices is superior to a matrix of one single structure [38]. According to the RIP condition of very sparse projection matrix, the correlation between very sparse projection vectors and the unit vectors can be controlled in a restricted range with high probability. Thus, the combination of the unit matrix and very sparse projection matrix inherits the property of each single matrix. Since both the unit diagonal matrix and very sparse projection matrix satisfy RIP, the combination of these matrices also satisfies RIP. Therefore, a new double-structure measurement matrix $\Phi \in \mathbb{R}^{M \times N}$ is designed by combining a part of unit diagonal matrix $\Phi_{3} \in \mathbb{R}^{M \times(N-M)}$ with $\Phi_{2}$, that is,

$$
\Phi=\left[\begin{array}{ll}
\Phi_{3} & \Phi_{2}
\end{array}\right]
$$

In order to verify the advantage of the proposed measurement matrix, the memory storage cost of the proposed matrix and Gaussian matrix is compared in Table 1. It is seen that the proposed matrix occupies a much smaller memory storage than Gaussian matrix of the same scale. Since the proposed measurement matrix is sparse, its sampling rate is much lower than the Gaussian matrix under the same conditions. Thus, it provides convenience for dealing with high-dimensional signals, saving memory storage and facilitating hardware storage and implementation. Based on these, the measurement matrix $\Phi \in \mathbb{R}^{M \times N}$ shown in equation (13) is applied in this paper.

\section{Two-Step DOA Estimation of a Single Snapshot}

A novel two-step approach for DOA estimation of singlesnapshot signal will be presented and some notations in the previous section will be employed in this section.

4.1. The First Step: DOA Preestimation. In the preestimation step, the search space of interest $\Theta$ is divided into $L_{1}$ parts by the interval $\pi / \gamma_{1}$, where $\gamma_{1}$ is a predefined step factor and $L_{1}<L$. Thus, the parameter $L_{1}$ can be expressed as

$$
L_{1}=\operatorname{INT}\left(\frac{\theta_{b}-\theta_{e}}{\left(\pi / \gamma_{1}\right)}\right)+1
$$

where $\theta_{b}-\theta_{e}$ denotes the whole angular range in $\Theta$ and $\operatorname{INT}(F)$ denotes the largest integer that does not exceed the value $F$.

Then, the overcomplete orthogonal dictionary with a rough division is used to construct the sparse transform matrix $\Psi_{1} \in \mathbb{C}^{N \times L_{1}}$, that is,

$$
\boldsymbol{\Psi}_{1}=\left[\begin{array}{ccc}
1 & \ldots & 1 \\
e^{-j 2 \pi(d / \lambda) \sin \bar{\varphi}_{1}} & \ldots & e^{-j 2 \pi(d / \lambda) \sin \bar{\varphi}_{L_{1}}} \\
\ldots & \ldots & \ldots \\
e^{-j(N-1) 2 \pi(d / \lambda) \sin \bar{\varphi}_{1}} & \ldots & e^{-j(N-1) 2 \pi(d / \lambda) \sin \bar{\varphi}_{L_{1}}}
\end{array}\right] .
$$


TABLE 1: Memory cost of the proposed matrix and Gaussian matrix.

\begin{tabular}{cccc}
\hline$M$ & $N$ & Proposed matrix (bytes) & Gaussian matrix (bytes) \\
\hline 10 & 40 & 1128 & 3200 \\
20 & 40 & 3000 & 6400 \\
30 & 40 & 4520 & 9600 \\
40 & 40 & 6600 & 12800 \\
\hline
\end{tabular}

Thus, the signal can be expressed as

$$
\mathbf{x}=\Psi_{1} \mathbf{y}_{1}+\mathbf{w},
$$

where $\mathbf{y}_{1} \in \mathbb{C}^{L_{1} \times 1}$ denotes the sparse representation of the estimated angle in a rough manner and $\bar{\varphi}_{j}=\theta_{b}+$ $\left(j-1 / L_{1}\right) \times\left(\theta_{e}-\theta_{b}\right)\left(j=1,2, \ldots L_{1}\right)$ denotes the $j^{\text {th }}$ angle in the search space $\Theta$.

Finally, the observed signals $\mathbf{s}_{1} \in \mathbb{C}^{M_{1} \times 1}$ are obtained by projecting the signal $\mathbf{x}$ into the double-structure measurement matrix $\Phi_{I}$, that is,

$$
\mathbf{s}_{1}=\Phi_{\mathbf{I}} \mathbf{x}=\Phi_{\mathbf{I}}\left(\Psi_{1} \mathbf{y}_{1}+\mathbf{w}\right),
$$

where $\Phi_{\mathrm{I}} \in \mathbb{R}^{M_{1} \times N}$ is constructed by using equation (13). Here, $M_{1}$ denotes the measurement number in the first step.

After the sparse representation of spatial signal, the rough DOA estimator $\left[\varphi_{1}, \varphi_{2}, \ldots, \varphi_{K}\right]$ is obtained using the OMP method. Here, $\varphi_{k}$ is the $k^{\text {th }}$ angle solution in the first step and $K$ is the sparse degree. In general, the sparse degree is the same as the number of actual angles.

4.2. The Second Step: Accurate DOA Estimation. In the second step, the search space of interest is narrowed as $\widehat{\Theta}$, which is generated adaptively according to the rough estimator $\left[\varphi_{1}, \varphi_{2}, \ldots, \varphi_{K}\right]$, which is

$$
\widehat{\Theta}=\left[\widehat{\Theta}_{1}, \widehat{\Theta}_{2}, \ldots, \widehat{\Theta}_{K}\right],
$$

with

$$
\widehat{\Theta}_{i}=\left(\varphi_{i}-\Delta \varphi_{i}, \varphi_{i}+\Delta \varphi_{i}\right), \quad i=1,2, \ldots, K,
$$

where $\widehat{\Theta}_{i}$ is a neighborhood area with the center $\varphi_{i}$ and radius $\Delta \varphi_{i}$.

Similar to the first step, the search space of interest $\widehat{\Theta}$ is divided into $L_{2}$ parts by the interval $\pi / \gamma_{2}$, where $\gamma_{2}$ is a predefined step factor. Thus, the parameter $L_{2}$ can be expressed as

$$
L_{2}=\operatorname{INT}\left(\frac{\operatorname{span}(\widehat{\Theta})}{\pi / \gamma_{2}}\right)+1
$$

Here, span means the difference between the maximum and minimum of the search space of interest. Note that the predefined step factor is larger than that in the first step, i.e., $\gamma_{2}>\gamma_{1}$, because the divided parts of the second step are smaller than those of the first step.

Then, the search space $\widehat{\Theta}$ is subdivided into $L_{2}$ parts to construct overcomplete matrix $\Psi_{2} \in \mathbb{R}^{N \times L_{2}}$, that is,

$$
\Psi_{2}=\left[\begin{array}{ccc}
1 & \cdots & 1 \\
e^{-j 2 \pi(d / \lambda) \sin \bar{\phi}_{1}} & \cdots & e^{-j 2 \pi(d / \lambda) \sin \bar{\phi}_{L_{2}}} \\
\ldots & \cdots & \cdots \\
e^{-j(N-1) 2 \pi(d / \lambda) \sin \bar{\phi}_{1}} & \cdots & e^{-j(N-1) 2 \pi(d / \lambda) \sin \bar{\phi}_{L_{2}}}
\end{array}\right] .
$$

Next, the signal can be expressed based on the new sparse base matrix as

$$
\mathbf{x}=\Psi_{2} \mathbf{y}_{2}+\mathbf{w},
$$

where $\mathbf{y}_{2} \in \mathbb{C}^{L_{2} \times 1}$ denotes the sparse representation of the accurate estimated angle and $\bar{\phi}_{j}=\min (\Theta)+\left(j-1 / L_{2}\right) \times$ $\operatorname{span}(\Theta)\left(j=1,2, \ldots L_{2}\right)$ denotes the $j^{\text {th }}$ angle in the search space $\widehat{\Theta}$.

Finally, the observed signals $\mathbf{s}_{2} \in \mathbb{C}^{M_{2} \times 1}$ are obtained by projecting the signal $\mathbf{x}$ into the double-structure measurement matrix $\Phi_{\mathrm{II}}$, that is,

$$
\mathbf{s}_{2}=\Phi_{\mathrm{II}} \mathbf{x}=\Phi_{\mathrm{II}}\left(\Psi_{2} \mathbf{y}_{2}+\mathbf{w}\right),
$$

where $\Phi_{\mathrm{II}} \in \mathbb{R}^{M_{2} \times N}$ is constructed by equation (13). Here, $M_{2}$ denotes the measurement number in the second step. Based on the new sparse representation of spatial signal, the accurate DOA estimator $\left[\phi_{1}, \phi_{2}, \ldots, \phi_{K}\right]$ is obtained using the OMP method, where $\phi_{k}$ is the $k^{\text {th }}$ accurate solution.

The flowchart of the proposed two-step DOA estimation algorithm is shown in Algorithm 2.

\section{Experiment Simulations and Discussion}

In the simulation experiments, we investigate the feasibility and accuracy of the proposed method for the single-snapshot DOA estimation, in terms of different measurement numbers, SNRs, and sparse degrees, respectively. The proposed method is compared with the general CS-based single-snapshot DOA estimation method, which is called the single-step Gaussian matrix method. In order to verify the effectiveness of the measurement matrix we used, it is also compared with the general Gaussian measurement matrix in the two-step scheme, which is called the two-step Gaussian matrix method. The Monte Carlo method is adopted to evaluate the estimation performance. Root mean square error (RMSE) is used to evaluate the accuracy of DOA estimation, which is described as below.

$$
\mathrm{RMSE}=\frac{1}{K} \sum_{k=1}^{K} \sqrt{\frac{1}{\mathrm{CNT}} \sum_{\mathrm{cnt}=1}^{\mathrm{CNT}}\left(\phi_{k, \mathrm{cnt}}-\theta_{k}\right)^{2}},
$$

where CNT is the number of Monte Carlo loops, $K$ is the sparse degree, $\phi_{k, \text { nnt }}$ is the estimated value of $k^{\text {th }}$ angle in the $\mathrm{cnt}^{\text {th }}$ Monte Carlo loop, and $\theta_{k}$ is the actual value of the $k^{\text {th }}$ angle. Thus, the RMSE value is measured by degree.

The parameters are defined as follows: the number of array antenna $N=40, d=\lambda / 2$, the sparse degree $K=4$, and $\mathrm{SNR}=15$. Note that though the parameter $K$ is assumed to be known, it is not required to be exactly the same as the number of sources in practice. The parameters 
Input:

The observed single-snapshot signal $\mathbf{x} \in \mathbb{C}^{N \times 1}$;

A new double-structure measurement matrix for preestimation, $\Phi_{\mathrm{I}} \in \mathbb{R}^{M_{1} \times N}$;

A new double-structure measurement matrix for the second step, $\Phi_{\mathrm{II}} \in \mathbb{R}^{M_{2} \times N}$; Output:

The DOA estimator, $\left[\phi_{1}, \phi_{2}, \ldots, \phi_{K}\right]$;

(1) Divide the initial search space $\Theta$ into $L_{1}\left(L_{1}<L\right)$ parts to construct the overcomplete dictionary matrix $\Psi_{1} \in \mathbb{C}^{N \times L_{1}}$.

(2) Generate the measured signal $\mathbf{z}_{1}=\Phi_{\mathrm{I}} \mathbf{x}$ and the sensing matrix $\mathbf{T}_{1}=\Phi_{\mathrm{I}} \Psi_{1}$.

(3) Calculate the DOA rough estimator $\left[\varphi_{1}, \varphi_{2}, \ldots, \varphi_{K}\right]$ using the OMP method [40].

(4) Narrow the search space as $\widehat{\Theta}=\left[\widehat{\Theta}_{1}, \widehat{\Theta}_{2}, \ldots, \widehat{\Theta}_{K}\right]$ based on the rough estimator, where $\widehat{\Theta}_{i}=\left(\varphi_{i}-\Delta \varphi_{i}, \varphi_{i}+\Delta \varphi_{i}\right) i=1,2, \ldots, K$.

(5) Divide the search space $\widehat{\Theta}$ into $L_{2}$ parts to construct the overcomplete dictionary matrix $\Psi_{2} \in \mathbb{C}^{N \times L_{2}}$.

(6) Generate the measured signal $\mathbf{z}_{2}=\Phi_{\mathrm{II}} \mathbf{x}$ and the sensing matrix $\mathbf{T}_{2}=\Phi_{\mathrm{II}} \Psi_{2}$.

(7) Calculate the DOA accurate estimator $\left[\phi_{1}, \phi_{2}, \ldots, \phi_{K}\right]$ using the OMP method in [40].

(8) return $\left[\phi_{1}, \phi_{2}, \ldots, \phi_{K}\right]$.

Algorithm 2: The proposed two-step CS-based algorithm.

in the two-step CS-based method are defined as follows: the search space of interest $\Theta$ is $\left[-90^{\circ}, 90^{\circ}\right]$, the step factor for preestimation $\gamma_{1}=30$, and the step factor for accurate estimation. $\gamma_{2}=100$ The 100 Monte Carlo loops are realized. As we found, two closely spaced DOAs, i.e., $1^{\circ}$ and $2^{\circ}$, are generally estimated by mistake as one DOA by MUSIC algorithm due to high correlation between two closely spaced sources, as shown in Figure 1. Here, the correlation coefficient between two closely spaced DOAs is 0.9407 . To verify the reliability of the proposed method in solving such estimation problem of highly correlated signals between each pair of sources, the actual angle values used in this experiment are randomly generated as $\theta=\left[\begin{array}{llll}-20^{\circ} & 1^{\circ} & 2^{\circ} & 35^{\circ}\end{array}\right]$. The radius $\Delta \varphi_{i}$ is chosen as $5^{\circ}$.

In Figure 2, the relationship between measurement number $M_{2}$ and RMSE is presented when $M_{1}=21$ (Figure 2(a)) and when $M_{1}=25$ (Figure 2(b)). It is seen that RMSE in Figure 2(b) is lower than that in Figure 2(a) because when $M_{1}$ increased, estimation accuracy of the first step was improved, which enhanced the accuracy of the final accurate estimation. When the measurement numbers $M_{1}$ and $M_{2}$ are increased, the RMSE of the proposed method is reduced. Such error mainly comes from the estimation process of $1^{\circ}$ and $2^{\circ}$. Thus, for the dense spatial signal estimation in a linear array system, the proposed algorithm can use a limited number of measurements to accurately estimate spatial signals that are spaced closer than the general resolution.

Figure 3 shows the RMSE performance with different SNRs. Here, the parameters of $N$ and $K$ are defined the same as the experiment mentioned above. The parameters of different CS-based methods are defined as follows. The measurement number in each step is defined as 21. The search space of interest $\Theta$ is $\left[-90^{\circ}, 90^{\circ}\right]$. In the two-step CS-based methods, the step factor for preestimation $\gamma_{1}=30$ and the step factor for accurate estimation $\gamma_{2}=100$. In the one-step CS-based method, the step factor is defined as 100. It is seen that the proposed method asymptotically follows CRB and performs better than other methods. Even when the SNR is lower than $0 \mathrm{~dB}$, the proposed method can obtain a lower RMSE.

Table 2 shows the average estimation results of 100 Monte Carlo trials when the sparse degrees are set as $k=3,4,5,6$, respectively. Here, the measurement numbers

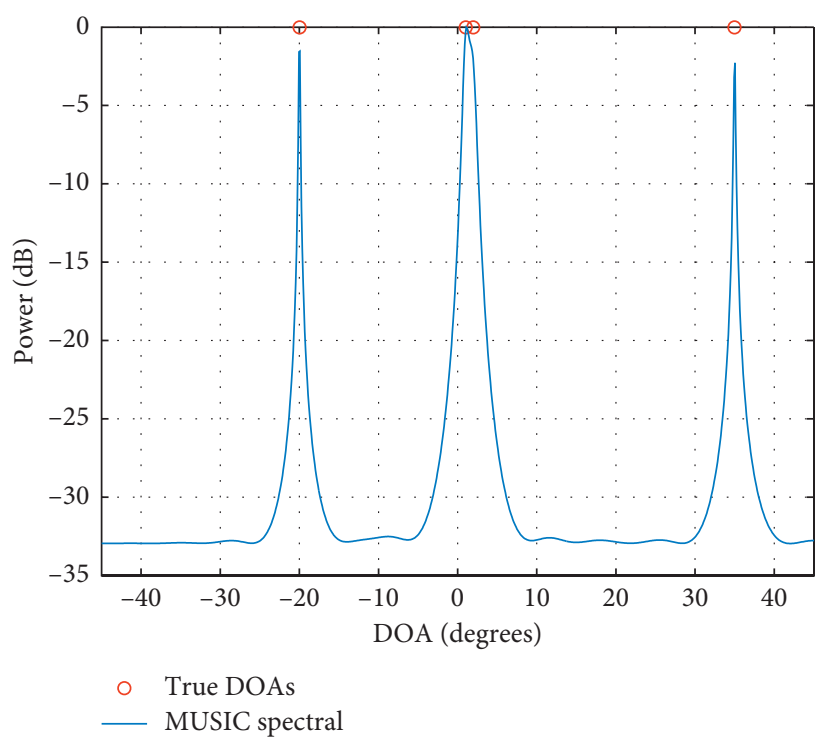

FIGURE 1: DOA estimation results using MUSIC algorithm.

are defined as $M_{1}=21, M_{2}=35$. It is seen that the estimated DOAs of the proposed method can distinguish the dense signals emitted from $1^{\circ}$ and $2^{\circ}$ with a higher accuracy. In order to evaluate the estimation accuracy more clearly, Figure 4 indicates the relationship between $M_{2}$ and RMSE under different sparse degree conditions. In Figure 4(a), $K=4$, the actual angle values $\theta=\left[\begin{array}{llll}-20^{\circ} & 1^{\circ} & 2^{\circ} & 35^{\circ}\end{array}\right]$. In Figure $4(\mathrm{~b}), \quad K=5$, the actual angle values $\theta=\left[\begin{array}{lllll}-40^{\circ} & -20^{\circ} & 1^{\circ} & 2^{\circ} & 35^{\circ}\end{array}\right]$. It is shown that the proposed two-step method performs better than others under different degrees of sparsity.

5.1. Comparison between One-Step and Two-Step DOA Estimation. Among the above experiments, the single-step CS approach and two-step CS approach are compared to analyze the effectiveness of two-step scheme. According to Figure 2, it is observed that the RMSE results obtained by two-step estimation methods are smaller than those of single-step estimation method under the same measurement 


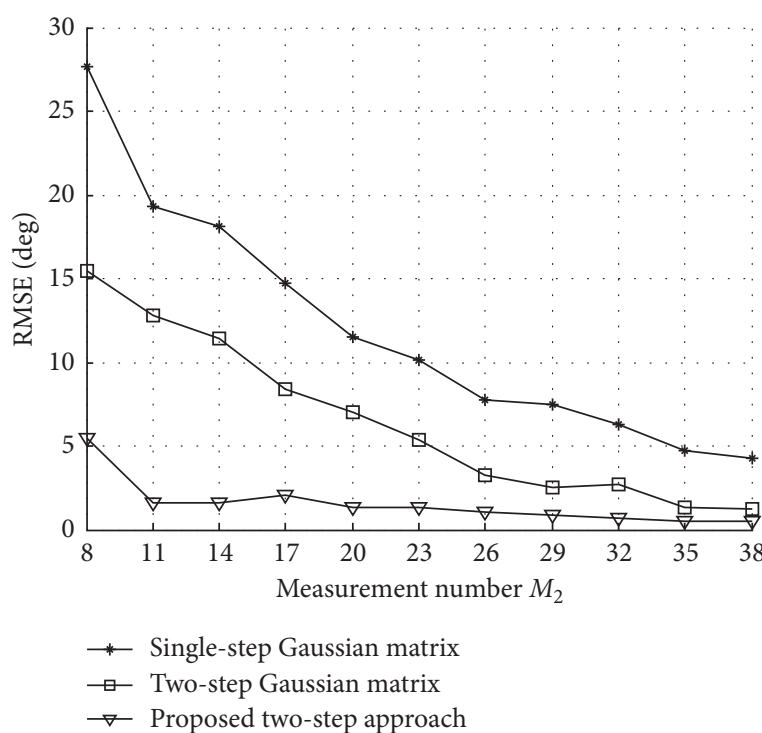

(a)

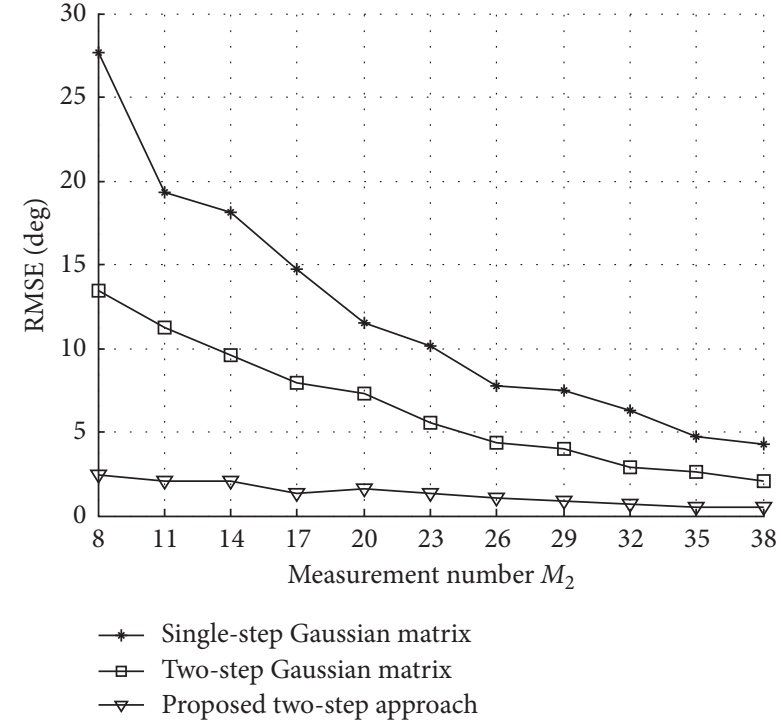

(b)

Figure 2: Relationship between RMSE and $M_{2}$ when (a) $M_{1}=21$ and (b) $M_{1}=25$.

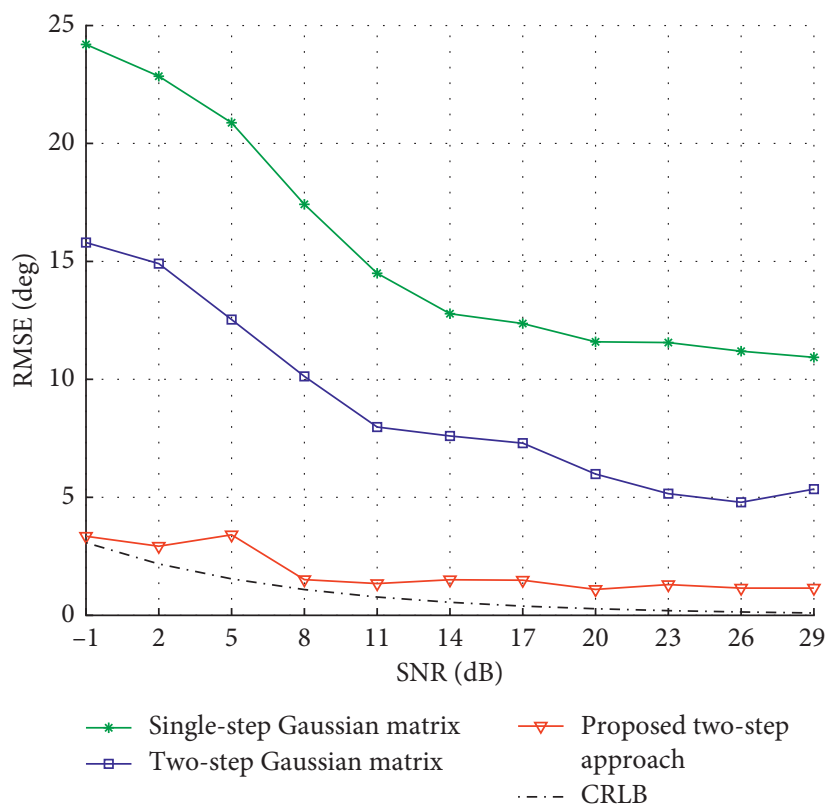

FIGURE 3: Relationship between RMSE and SNR.

number condition. Figure 3 shows that under the same SNR condition, the two-step estimation methods are superior to the single-step estimation method. For the cases with different degrees of sparsity, Table 2 and Figure 4 indicate that compared with the single-step method, the two-step estimation methods can reduce the estimated error caused by the dense signals emitted from $1^{\circ}$ and $2^{\circ}$.

\subsection{Discussion about Measurement Matrix Construction.} Additionally, Figures 2-4 present the comparison results of the two-step CS approaches with different measurement matrices. It is found that estimation accuracy of the proposed double-structure matrix is better than that of Gaussian matrix, which verifies the reliability of the proposed double-structure measurement matrix. Considering that the estimation results of the first step determined the search space of the second step in the CS-based approach, different measurement matrices in the first step would affect the final estimation results. Thus, we investigate the performance of different measurement matrices in the first step for dense DOA estimation. In the experiment, two closely spaced DOAs are considered for estimation, i.e., $\theta=\left[\begin{array}{ll}1^{\circ} & 2^{\circ}\end{array}\right]$. The proposed measurement matrix and Gaussian matrix are used in the first step, respectively, and the same second step is used as in the proposed algorithm. 
TABLE 2: Comparison of average DOA estimation results using various methods.

\begin{tabular}{|c|c|c|c|c|}
\hline \multirow{2}{*}{ Sparsity } & \multirow{2}{*}{ True angles } & \multicolumn{3}{|c|}{ Estimated angles } \\
\hline & & Proposed & 2-step Gaussian & 1-step \\
\hline \multirow{3}{*}{$k=3$} & $1^{\circ}$ & $0.6^{\circ}$ & $0.5910^{\circ}$ & $-1.7^{\circ}$ \\
\hline & $2^{\circ}$ & $2.4^{\circ}$ & $2.544^{\circ}$ & $12.4^{\circ}$ \\
\hline & $35^{\circ}$ & $34.8^{\circ}$ & $34.935^{\circ}$ & $36.6^{\circ}$ \\
\hline \multirow{4}{*}{$k=4$} & $-20^{\circ}$ & $-21^{\circ}$ & $-20.478^{\circ}$ & $-22.347^{\circ}$ \\
\hline & $1^{\circ}$ & $0^{\circ}$ & $-12.501^{\circ}$ & $-1.287^{\circ}$ \\
\hline & $2^{\circ}$ & $1.8^{\circ}$ & $2.295^{\circ}$ & $10.674^{\circ}$ \\
\hline & $35^{\circ}$ & $34.8^{\circ}$ & $34.854^{\circ}$ & $35.514^{\circ}$ \\
\hline \multirow{5}{*}{$k=5$} & $-40^{\circ}$ & $-39^{\circ}$ & $-38.82^{\circ}$ & $-42.228^{\circ}$ \\
\hline & $-20^{\circ}$ & $-19.2^{\circ}$ & $-22.422^{\circ}$ & $-22.896^{\circ}$ \\
\hline & $1^{\circ}$ & $0^{\circ}$ & $-13.113^{\circ}$ & $-2.799^{\circ}$ \\
\hline & $2^{\circ}$ & $1.8^{\circ}$ & $2.928^{\circ}$ & $11.16^{\circ}$ \\
\hline & $35^{\circ}$ & $34.8^{\circ}$ & $34.89^{\circ}$ & $36.423^{\circ}$ \\
\hline \multirow{6}{*}{$k=6$} & $-40^{\circ}$ & $-39^{\circ}$ & $-38.91^{\circ}$ & $35.514^{\circ}$ \\
\hline & $-20^{\circ}$ & $-19.2^{\circ}$ & $-22.395^{\circ}$ & $-22.797^{\circ}$ \\
\hline & $1^{\circ}$ & $0^{\circ}$ & $-12.591^{\circ}$ & $-2.628^{\circ}$ \\
\hline & $2^{\circ}$ & $1.8^{\circ}$ & $3.102^{\circ}$ & $11.583^{\circ}$ \\
\hline & $35^{\circ}$ & $34.8^{\circ}$ & $34.899^{\circ}$ & $37.449^{\circ}$ \\
\hline & $70^{\circ}$ & $70.8^{\circ}$ & $69.999^{\circ}$ & $70.299^{\circ}$ \\
\hline
\end{tabular}

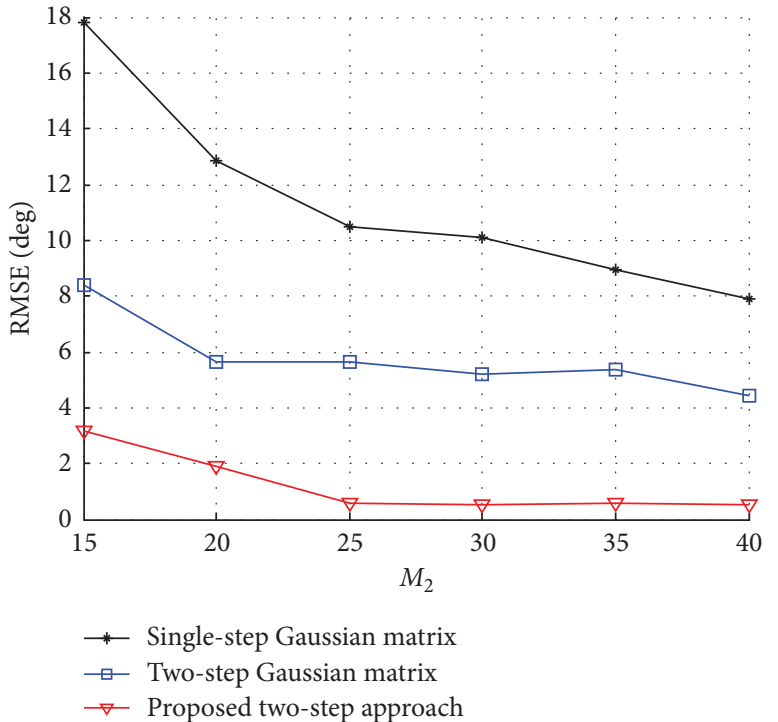

(a)

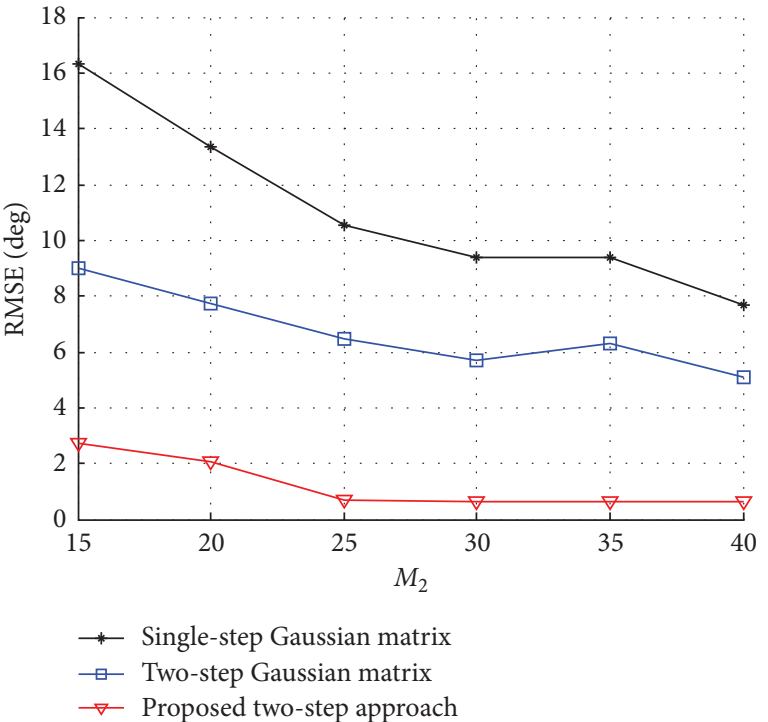

(b)

FIgURE 4: Relationship between RMSE and $M_{2}$ when (a) $k=4$ and (b) $k=5$.

The comparison results are plotted in Figure 5. It is found that the proposed algorithm can obtain a better RMSE than Gaussian matrix for dense DOA estimation. The reason is that in the first CS-based procedure, the proposed measurement matrix can obtain a better preestimator with higher accuracy than Gaussian matrix. The better preestimator is helpful to construct a better search range $\widehat{\Theta}$ for the second step and then generate a better accurate estimator. Therefore, it is efficient to apply the double-structure measurement matrix to two-step CS-based approach to improve the estimation accuracy.
5.3. Comparison with Other Single-Snapshot DOA Estimation Methods for Dense DOA Estimation. Finally, the proposed method is compared with other existing single-snapshot DOA estimation methods. Considering that the latest single-snapshot DOA estimation methods are mainly designed based on the CS theory, especially some off-grid CS-based methods might solve the problem of dense parameter estimation. So, we compared the proposed method with two off-grid CS-based approaches, that is, the OGSBI method [15] and the SAMV method [14]. In this experiment, $N=40$, a single-snapshot signal is observed from 


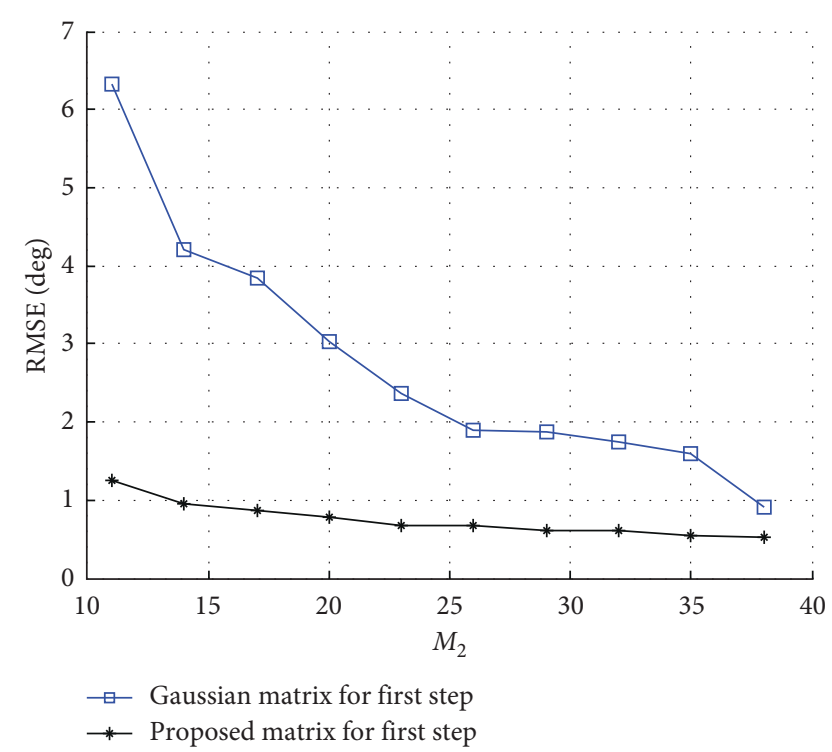

FIgURE 5: Comparison of two different measurement matrices in the first step in terms of $M_{2}$ and RMSE with two densely distributed signals.

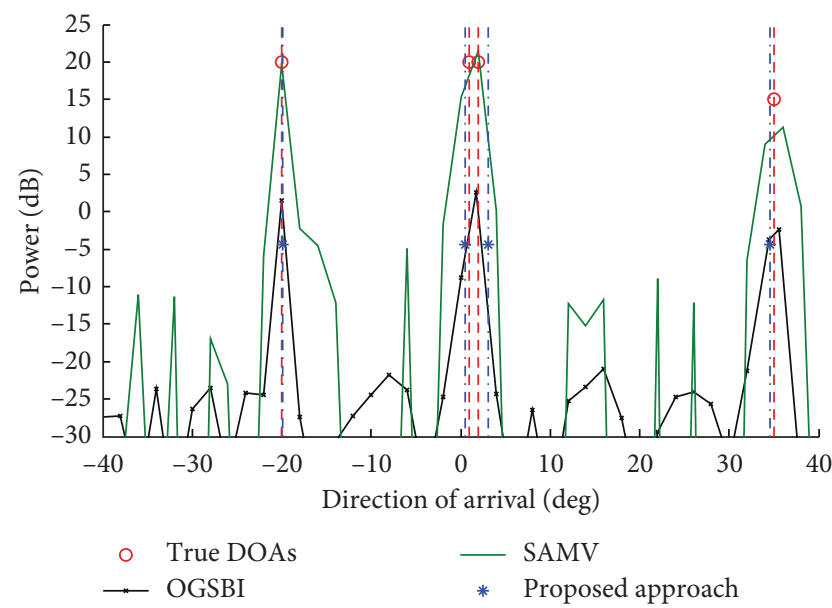

Figure 6: Comparison results of OGSBI and SAMV with the proposed approach.

four angles $\theta=\left[\begin{array}{llll}-20^{\circ} & 1^{\circ} & 2^{\circ} & 35^{\circ}\end{array}\right]$. In order to make the comparative experiment fair, the grid spacing of OGSBI and SAMV is chosen as $2^{\circ}$. The number of Monte Carlo loops is 50. Figure 6 shows one simulation trail of the Monte Carlo experiments to compare the estimated DOAs of three methods. It is seen that OGSBI and SAMV fail to distinguish two peaks when estimating two dense DOAs. In other words, in this experiment, the proposed method can successfully estimate four DOAs, but the OGSBI and SAMV methods can only obtain three DOAs because two dense DOAs are misunderstood as a single DOA. In brief, there is usually a problem of high computational complexity while existing estimation approaches achieve higher accuracy for dense DOA estimation. In our calculations, the average time costs using the same Monte
Carlo loop but three different methods in the same example are recorded. The results show that the average execution times are around 3 seconds, 0.01 seconds, and 0.3 seconds when using OGSBI, SAMV, and the proposed methods, respectively. Though SAMV could achieve the solution with a lower time cost, its estimation accuracy is poor. Both the OGSBI method and the proposed method require a certain time cost to deal with grid updating process. However, compared to OGSBI, the proposed method costs less time.

\section{Conclusion}

In this paper, a two-step algorithm based on CS theory is proposed to solve the single-snapshot dense DOA estimation problem. The features of the proposed method include the following: (1) the search spaces with coarse and refined grids are used in the two estimations, respectively, so that the size of the overcomplete matrices is reduced to a low level; (2) a new double-structure measurement matrix is firstly presented using very sparse projection scheme and chaotic sequence matrix; and (3) the performance of the proposed algorithm is quite good demonstrated by numerical examples.

It is shown that the proposed double-structure measurement matrix costs less memory than pure chaotic matrix, which performs better than single-step and two-step Gaussian method, and it can work well with smaller measurement numbers and lower SNR. It is also shown that the proposed method performs better for single-snapshot DOA estimation of high correlation signals, when compared with some existing off-grid DOA estimation methods (OGSBI and SAMV). It should be noted that the number of array elements $N$ should be not smaller than the measurement number $M$ and the value of $M$ should meet the requirement of the CS theory, i.e., $M \geq K \times \log _{2}(N / K)$ in our proposed method.

In our future work, we will further analyze the performance of the combined methods of SVD and the proposed two-step CS algorithm for the low-altitude target dynamic detection, which is the key to automotive assistance driver system and so on.

\section{Data Availability}

The data used to support the findings of this study are included within the article.

\section{Conflicts of Interest}

The authors declare that they have no conflicts of interest.

\section{Acknowledgments}

This study was supported by the National Natural Science Foundation of China under grant nos. 61401145 and 61701306 and the Natural Science Foundation of Shanghai under grant no. 19ZR1437600. 


\section{References}

[1] C. Peng, Z. Cao, Z. Chen, and X. Wang, "Off-grid DOA estimation using sparse bayesian learning in MIMO radar with unknown mutual coupling," IEEE Transactions on Signal Processing, vol. 67, no. 1, pp. 208-220, 2019.

[2] A. Alexandridis and A. Mouchtaris, "Multiple sound source location estimation in wireless acoustic sensor networks using DOA estimates: the data-association problem," IEEE/ACM Transactions on Audio Speech \& Language Processing, vol. 26, no. 2, pp. 342-356, 2017.

[3] Q. Liu and X. Wang, "Direction of arrival estimation via reweighted 1 norm penalty algorithm for monostatic MIMO radar $l_{1}$ norm penalty algorithm for monostatic mimo radar," Multidimensional Systems and Signal Processing, vol. 29, no. 2, pp. 733-744, 2016.

[4] Q. Liu, Y. Gu, and H. C. So, "Doa estimation in impulsive noise via low-rank matrix approximation and weakly convex optimization," IEEE Transactions on Aerospace and Electronic Systems, vol. 55, no. 6, pp. 3603-3616, 2019.

[5] R. Schmidt, "Multiple emitter location and signal parameter estimation," IEEE Transactions on Antennas and Propagation, vol. 34, no. 3, pp. 276-280, 1986.

[6] J. Lin, X. Ma, S. Yan, and C. Hao, "Time-Frequency multi-invariance ESPRIT for DOA estimation," IEEE Antennas and Wireless Propagation Letters, vol. 15, pp. 770-773, 2016.

[7] P. Häcker and B. Yang, "Single snapshot DOA estimation," Advances in Radio Science, vol. 8, pp. 251-256, 2010.

[8] P. Rocca, M. A. Hannan, M. Salucci, and A. Massa, "Singlesnapshot DoA estimation in array antennas with mutual coupling through a multiscaling BCS strategy," IEEE Transactions on Antennas and Propagation, vol. 65, no. 6, pp. 3203-3213, 2017.

[9] X. Li, X. Ma, S. Yan, and C. Hou, "Single snapshot DOA estimation by compressive sampling," Applied Acoustics, vol. 74, no. 7, pp. 926-930, 2013.

[10] Q. Shen, W. Liu, W. Cui, and S. Wu, "Underdetermined DOA estimation under the compressive sensing framework: a review," IEEE Access, vol. 4, pp. 8865-8878, 2016.

[11] J. Zhu and M. A. Badiu, "Expectation propagation line spectral estimation," 2019.

[12] J. Zhu, L. Han, R. S. Blum, and Z. Xu, "Multi-snapshot Newtonized orthogonal matching pursuit for line spectrum estimation with multiple measurement vectors," Signal Processing, vol. 165, pp. 175-185, 2019.

[13] J. Zhu, Q. Zhang, P. Gerstoft, M.-A. Badiu, and Z. Xu, "Grid-less variational bayesian line spectral estimation with multiple measurement vectors," Signal Processing, vol. 161, pp. 155-164, 2019.

[14] H. Abeida, Q. Zhang, J. Li, and N. Merabtine, "Iterative sparse asymptotic minimum variance based approaches for array processing," IEEE Transactions on Signal Processing, vol. 61, no. 4, pp. 933-944, 2013.

[15] Z. Yang, L. Xie, and C. Zhang, "Off-grid direction of arrival estimation using sparse bayesian inference," IEEE Transactions on Signal Processing, vol. 61, no. 1, pp. 38-43, 2013.

[16] Q. Liu, H. C. So, and Y. Gu, "Off-grid doa estimation with nonconvex regularization via joint sparse representation," Signal Processing, vol. 140, pp. 171-176, 2017.

[17] H. Zhu, G. Leus, and G.B. Giannakis, "Sparsity-cognizant total least-squares for perturbed compressive sampling," IEEE Transactions on Signal Processing, vol. 59, no. 5, p. 2002, 2011.

[18] M. Carlin, P. Rocca, G. Oliveri, F. Viani, and A. Massa, "Directions-of-Arrival estimation through bayesian compressive sensing strategies," IEEE Transactions on Antennas and Propagation, vol. 61, no. 7, pp. 3828-3838, 2013.

[19] G. Li, Y. Gu, and Y. Gu, "Restricted Isometry property of Gaussian random projection for finite set of subspaces," IEEE Transactions on Signal Processing, vol. 66, no. 7, pp. 1705-1720, 2018.

[20] E. J. Candès, J. Romberg, and T. Tao, "Robust uncertainty principles: exact signal reconstruction from highly incomplete frequency information," IEEE Transactions on Information Theory, vol. 52, no. 2, pp. 489-509, 2006.

[21] E. J. Candes and T. Tao, "Near-optimal signal recovery from random projections: universal encoding strategies?" IEEE Transactions on Information Theory, vol. 52, no. 12, pp. 5406-5425, 2006.

[22] J. A. Tropp and A. C. Gilbert, "Signal recovery from random measurements via orthogonal matching pursuit," IEEE Transactions on Information Theory, vol. 53, no. 12, pp. 4655-4666, 2007.

[23] W. Shuang, C. Tao, W. Feng, and D. Jiang, "Performance of different measurement matrices of compressed sensing on sparse spatial spectral estimation," 2017.

[24] L. Yu, J. Barbot, G. Zheng, and H. Sun, "Compressive sensing with chaotic sequence," IEEE Signal Processing Letters, vol. 17, no. 8, pp. 731-734, 2010.

[25] V. Abolghasemi, S. Ferdowsi, and S. Sanei, "A gradient-based alternating minimization approach for optimization of the measurement matrix in compressive sensing," Signal Processing, vol. 92, no. 4, pp. 999-1009, 2012.

[26] W. Chen, M. R. D. Rodrigues, and I. J. Wassell, "Projection design for statistical compressive sensing: a tight frame based approach," IEEE Transactions on Signal Processing, vol. 61, no. 8, pp. 2016-2029, 2013.

[27] E. V. Tsiligianni, L. P. Kondi, and A. K. Katsaggelos, "Construction of incoherent unit norm tight frames with application to compressed sensing," IEEE Transactions on Information Theory, vol. 60, no. 4, pp. 2319-2330, 2014.

[28] C. Rusu and N. Gonz'alez-Prelcic, "Optimized compressed sensing via incoherent frames designed by convex optimization," 2015.

[29] H. Bai, S. Li, and X. He, "Sensing matrix optimization based on equiangular tight frames with consideration of sparse representation error," IEEE Transactions on Multimedia, vol. 18, no. 10, pp. 2040-2053, 2016.

[30] G. Li, Z. Zhu, D. Yang, L. Chang, and H. Bai, "On projection matrix optimization for compressive sensing systems," IEEE Transactions on Signal Processing, vol. 61, no. 11, pp. 28872898, 2013.

[31] G. Chen, D. Zhang, Q. Chen, and D. Zhou, "The characteristic of different chaotic sequences for compressive sensing," 2012.

[32] L. Ping, "Very sparse stable random projections for dimension reduction in $l_{\alpha}(0 \leq \alpha \leq 2)$ norm," 2007.

[33] H. Gan, S. Xiao, and Y. Zhao, "A novel secure data transmission scheme using chaotic compressed sensing," IEEE Access, vol. 6, pp. 4587-4598, 2018.

[34] H. L. V. Trees, Optimum Array Processing: Part IV of Detection, Estimation, and Modulation Theory, Wiley-Interscience, New York, NY, USA, 2002.

[35] H. Krim and M. Viberg, "Two decades of array signal processing research: the parametric approach," IEEE Signal Processing Magazine, vol. 13, no. 4, pp. 67-94, 1996.

[36] R. Cao, B. Liu, F. Gao, and X. Zhang, "A low-complex onesnapshot doa estimation algorithm with massive ula," IEEE Communications Letters, vol. 21, no. 5, pp. 1071-1074, 2017. 
[37] S. Fortunati, R. Grasso, F. Gini, M. Greco, and K. LePage, "Single-snapshot doa estimation by using compressed sensing," Eurasip Journal on Advances in Signal Processing, vol. 1, p. 120, 2014.

[38] Y. C. Eldar and G. Kutyniok, "Compressed sensing: theory and applications," Corr, vol. 52, no. 4, pp. 1289-1306, 2011.

[39] R. Wei, Q. Wang, and Z. Zhao, "Two-dimensional DOA estimation based on separable observation model utilizing weighted L1-norm penalty and bayesian compressive sensing strategy," 2017.

[40] T. T. Cai and L. Wang, "Orthogonal matching pursuit for sparse signal recovery with noise," IEEE Transactions on Information Theory, vol. 57, no. 7, pp. 4680-4688, 2011.

[41] F. Pourkamali-Anaraki and S. M. Hughes, "Memory and computation efficient PCA via very sparse random projections," 2014.

[42] F. Pourkamali-Anaraki, S. Becker, and S. M. Hughes, "Efficient dictionary learning via very sparse random projections," 2015.

[43] L. Ping, T. J. Hastie, and K. W. Church, "Very sparse random projections," 2006. 